\title{
AVALIAÇÃO ESTOCÁSTICA DO MODELO DE ADVECÇÃO-DIFUSÃO DE SUBSTÂNCIAS RADIOATIVAS LIBERADAS NA ATMOSFERA POR USINAS NUCLEARES
}

\author{
Guilherme J. Weymar ${ }^{1}$, Bardo E. J. Bodmann ${ }^{1}$, Marco T. M. B. Vilhena ${ }^{1}$, Daniela Buske ${ }^{2}$
}

\section{RESUMO}

Este artigo apresenta uma avaliação estocástica do modelo de advecção-difusão para a liberação de substâncias radioativas do experimento de Angra dos Reis. A função densidade probabilidade mostra a incompatibilidade da parametrização dos coeficientes turbulentos no problema.

\begin{abstract}
In this article, we present a stochastic evaluation of the advection-diffusion model for release of radioactive substances in the Angra dos Reis experiment. The probability density function indicates the incompatibility of the parameterization of turbulent coefficients in the problem.
\end{abstract}

\section{INTRODUÇÃO}

O uso apropriado dos modelos determinísticos de transporte e difusão na baixa atmosfera para simulação da concentração de poluentes deve ser baseado no estudo de sua capacidade de representar situações reais adequadamente, mesmo que a dispersão de poluentes seja um processo estocástico. Logo, este trabalho apresenta uma avaliação estocástica do modelo de dispersão de substâncias radioativas liberadas na atmosfera por usinas nucleares. Esta análise é conduzida com o modelo determinístico [1], para mostrar se está representando a essência do fenômeno da dispersão de substâncias radioativas que é de natureza estocástica. É apresentada a função densidade probabilidade com a finalidade de validar o modelo.

\section{METODOLOGIA}


Consideremos a equação de advecção-difusão tridimensional transiente que determina a concentração de um poluente radioativo:

$$
\frac{\partial \bar{C}}{\partial t}+\bar{u} \frac{\partial \bar{C}}{\partial x}+\bar{v} \frac{\partial \bar{C}}{\partial y}+\bar{w} \frac{\partial \bar{C}}{\partial z}=\frac{\partial}{\partial x}\left(K_{x} \frac{\partial \bar{C}}{\partial x}\right)+\frac{\partial}{\partial y}\left(K_{y} \frac{\partial \bar{C}}{\partial y}\right)+\frac{\partial}{\partial z}\left(K_{z} \frac{\partial \bar{C}}{\partial z}\right)-\lambda \bar{C}
$$

onde $\bar{C}$ é a concentração média do contaminante passivo $\left(\mathrm{g} / \mathrm{m}^{3}\right), \bar{u}, \bar{v}$ e $\bar{w}$ são os termos advectivos os ventos médios nas direções $x, y$ e $z . K_{x}, K_{y}$ e $K_{z}$ são os coeficientes de difusão turbulento $\left(\mathrm{m}^{2} / \mathrm{s}\right)$. A Eq. (1) está sujeita a condição inicial nula e as condições de contorno de fluxo zero nas fronteiras do domínio do problema, e a fonte é caracterizada por $\bar{u} C(0, y, z, t)=$ $Q \delta\left(y-y_{0}\right) \delta\left(z-H_{s}\right)$ em $x=0$. $Q$ é a taxa de emissão do poluente, $H_{s}$ é a altura da fonte, $\delta$ é a função delta de Dirac. Os detalhes da solução do modelo podem ser encontrado em [1].

O diagrama de espalhamento dos dados observados e preditos das concentrações ao nível do solo é mostrado na Fig. (1a). As concentrações preditas foram geradas pelo modelo 3D-GILTT [2] para os cinco experimentos de Angra dos Reis.

É criada uma distribuição, a partir das distâncias dos pontos do diagrama de espalhamento Fig. (1a) à reta bissetriz, decorrente da natureza estocástica. Com a distribuição de probabilidade, deduziu-se a função densidade probabilidade.

\section{RESULTADOS E CONCLUSÕES}

A Fig. (1b) mostra o gráfico da função densidade probabilidade, que apresenta uma distribuição estreita em torno das distâncias próximas de zero, superimposto por uma distribuição larga cujo máximo é deslocado. Observa-se que o modelo é compatível com uma parte do fenômeno, em torno de $30 \%$, na qual representa o pico que está centrado no eixo vertical, o outro pico deslocado é onde o modelo diverge das concentrações observadas.

Esta incompatibilidade é provavelmente devido à parametrização dos coeficientes turbulentos não adequados para as condições meteorológicas do problema, que não levam em conta a rugosidade complexa e a peculiaridade do terreno, isto é, proximidade de terra-mar, visto que a orografia da região Angra dos Reis é altamente complexo e próximo ao mar. 
a)

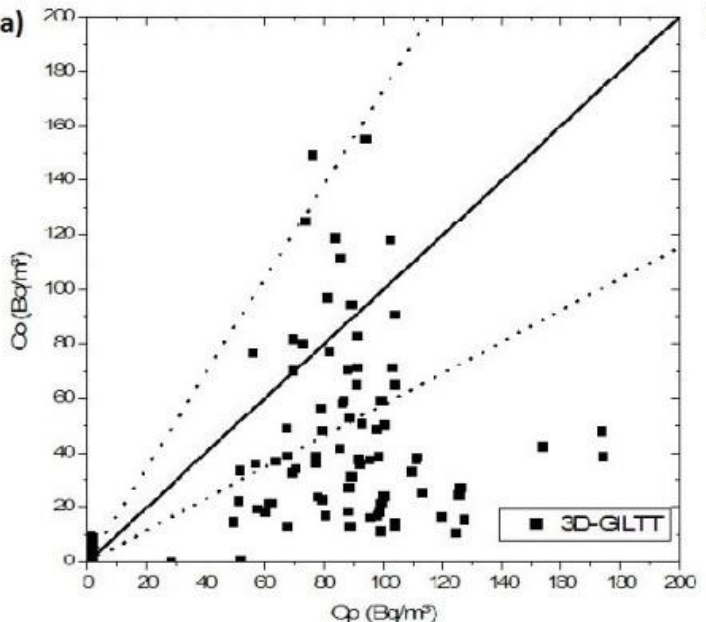

b)

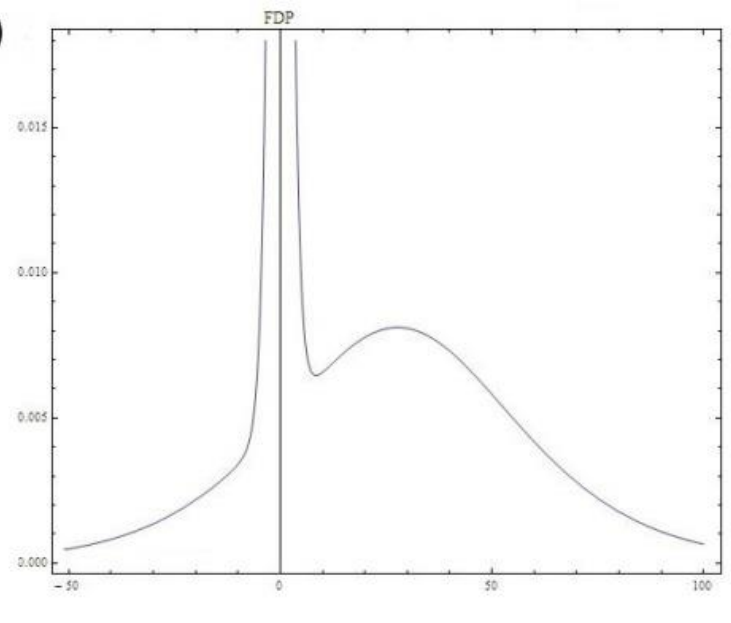

Figura 1: a) Diagrama de espalhamento dos dados preditos pela GILTT em comparação com os dados observados. b) Função Densidade Probabilidade.

Notou-se que em outros trabalhos o mesmo modelo determinístico se mostrou eficiente com a situação meteorológica adequadamente caracterizado pela parametrização, o que indica para o presente estudo que alterações nas parametrizações são necessárias para melhorar a acuidade do modelo. A Fig. (2) que mostra o nível em função do intervalo de confiança afirma este fato. Cumpre mencionar que o método exposto é eficiente para validar modelos.

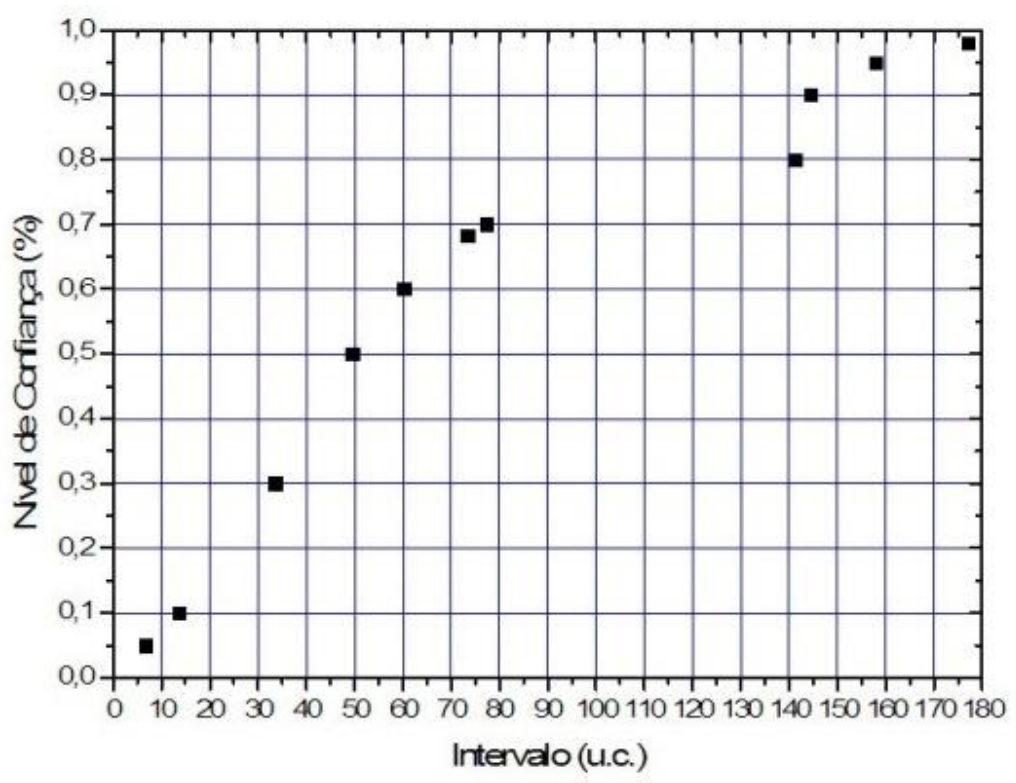

Figura 2: Nível de confiança versus intervalo de confiança.

\section{AGRADECIMENTOS}


À CAPES e CNPq pelo apoio financeiro.

\section{BIBLIOGRAFIA}

[1] Weymar, G. J., 2012. Simulação analítica da dispersão de substâncias radioativas liberadas na atmosfera por usinas nucleares pelo método GILTT. Dissertação de mestrado, Programa de pós-graduação em Engenharia Mecânica, UFRGS.

[2] Buske, D., Vilhena, M. T., Moreira, D. M., Tirabassi, T., 2010. An Analytical Solution for the Transient Two-dimensional Advective-Diffusion Equation with Non-Fickian Closure in Cartesian Geometry by the General Integral Transform Technique. Vol. 2, pag. 33-40. Integral Methods in Science and Engineering: Techniques and Applications. 